\title{
Diagnostic Utility of Performing Flow Cytometry on Provider-Submitted Endoscopically Collected Gastrointestinal Samples
}

\author{
Regina M. Plummer, Amy K. Beckman, Meghan M. Hupp, Elizabeth L. Courville, \\ Sarah A. Williams, Michael A. Linden
}

Division of Hematopathology, Department of Laboratory Medicine and Pathology, University of Minnesota, Minneapolis, MN, USA

Email: plumm035@umn.edu

How to cite this paper: Plummer, R.M., Beckman, A.K., Hupp, M.M., Courville, E.L., Williams, S.A. and Linden, M.A. (2020) Diagnostic Utility of Performing Flow Cytometry on Provider-Submitted Endoscopically Collected Gastrointestinal Samples. Open Journal of Blood Diseases, 10, 1-11. https://doi.org/10.4236/ojbd.2020.101001

Received: January 7, 2020

Accepted: January 28, 2020

Published: January 31, 2020

Copyright $\odot 2020$ by author(s) and Scientific Research Publishing Inc. This work is licensed under the Creative Commons Attribution International License (CC BY 4.0). http://creativecommons.org/licenses/by/4.0/

\begin{abstract}
Multicolor flow cytometry (MFC) is essential to the diagnosis of non-Hodgkin lymphoma (NHL). In our institution, MFC specimens are submitted by pathologists or an ordering provider. As endoscopy has revolutionized the ability to biopsy the gastrointestinal (GI) tract, our lab increasingly receives provider-submitted, endoscopically-acquired GI biopsies (PEGIB) for MFC analysis. This study evaluates the clinical utility of MFC performed on PEGIB and proposes a new testing algorithm to enhance the pathology team's role in MFC test utilization. Fifty-five archival PEGIB MFC cases were identified and histories were reviewed. MFC was non-contributory to the overall diagnosis in 85\% of PEGIB. Retroactively implementing an algorithm that used PEGIB permanent section screening to triage the 55 archival cases resulted in the appropriate identification of $100 \%$ of specimens whose diagnosis would have benefitted from MFC analysis, and the optimization of test utilization by decreasing unnecessary MFC studies.
\end{abstract}

\section{Keywords}

Flow Cytometry, Endoscopic Biopsies, GI Biopsies, Endoscopy, Quality Improvement

\section{Introduction}

Multicolor flow cytometry (MFC) is an essential component to the diagnosis of non-Hodgkin lymphoma (NHL). In our institution, material submitted for MFC 
may be submitted by a pathologist or an ordering provider. While most specimens received for MFC analysis at our institution are from hematolymphoid specimens such as bone marrow, peripheral blood, or lymph node, we are seeing an increase in non-hematolymphoid tissue specimens. Among these, ordering provider-submitted, endoscopically-acquired gastrointestinal biopsies (PEGIB) caught the attention of the hematopathology staff due to the inability to triage them in a similar manner as hematolymphoid tissues.

Traditionally, tissue specimens on which MFC is ordered are managed according to our institution's lymphoma protocol. During a lymphoma protocol, touch preparations are made from fresh tissue. One touch preparation is stained using Diff-Quik to ascertain adequacy and preliminary morphology, while the others are left unstained for possible fluorescent in situ hybridization (FISH) testing. If sufficient hematolymphoid tissue is identified on the stained touch preparation slide, the specimen is divided and submitted for MFC analysis, potential cytogenetic testing, and morphologic examination.

However, endoscopically-acquired GI biopsies are typically not suitable for high-quality touch imprints, presumably due to their mucinous nature. Moreover, a lymphocyte predominant inflammatory infiltrate is part of both normal histology as well as a feature of a variety of non-lymphomatous pathologic entities within the GI tract. To add to this, the gastroenterologists at our institution often submit multiple biopsies from different locations from within the GI tract from a single endoscopic examination, multiplying the cost of testing since each sample is processed and analyzed as a separate specimen. Therefore, we felt it necessary to review the practice of PEGIB MFC specimens.

\section{Methods}

This study was reviewed by the University of Minnesota Institutional Review Board (IRB), determined to involve no greater than minimal risk, and therefore, a formal consent process for participation was waived. This study consisted of two phases. For the first phase, adult MFC cases performed on PEGIB specimens collected between 2012 and 2017 were identified using natural language searches in Sunquest CoPathPlus ${ }^{\mathrm{TM}}$ (version 6.1.1) database, our department's anatomic pathology information system. We searched MFC cases for the following terms: esophagus, stomach, duodenum, jejunum, ileum, colon, rectum, small bowel, large bowel, small intestine, and large intestine. Non-endoscopically acquired biopsies (open biopsies), excision/resection specimens, and specimens from patients under the age of 18 were excluded. For each case, we reviewed the patient's medical and pathology records and noted the associated microscopic diagnoses, patient history, procedure indication, and relevant endoscopic and imaging findings to identify possible clinical parameters that correlated with MFC results. A two-tailed Fisher's exact test was used to compare categorical data.

In the second phase of the study, we proposed and retrospectively applied a new testing algorithm meant to enhance the pathology team's role in MFC test 
utilization (Figure 1). Generally, at our institution, if endoscopists submit a specimen for MFC, they take a second biopsy from the same site for morphologic assessment. We have a 24 hour turnaround time on the majority of GI biopsies at our institution, such that permanent hematoxylin and eosin (H\&E) stained slides are typically reviewed by a pathologist and signed out within 24 hours. The new algorithm proposed holding endoscopically acquired MFC specimens for up to 24 hours in RPMI under refrigeration at $4^{\circ}-8^{\circ}$ Celsius while permanent slides are prepared from the biopsy specimens. Although fresher specimens have higher cellular yields, it is generally accepted that specimens tested within 24 hours of collection stored under proper conditions maintain acceptable cellular viability [1] [2].

During the holding period, a member of the hematopathology staff (either a hematopathologist or senior resident) would review the permanent sections associated with each PEGIB MFC specimen, and then choose to cancel or move forward with the MFC order based upon their morphologic assessment. This method of triaging is currently practiced by our cytopathology division on fine needle aspiration (FNA) specimens when the need for MFC is uncertain prior to morphologic assessment. This practice produces no significant delays in diagnostic turnaround time or alterations in specimen cellular viability of any known consequence.

To test our proposed algorithm, we pulled and de-identified the H\&E stained

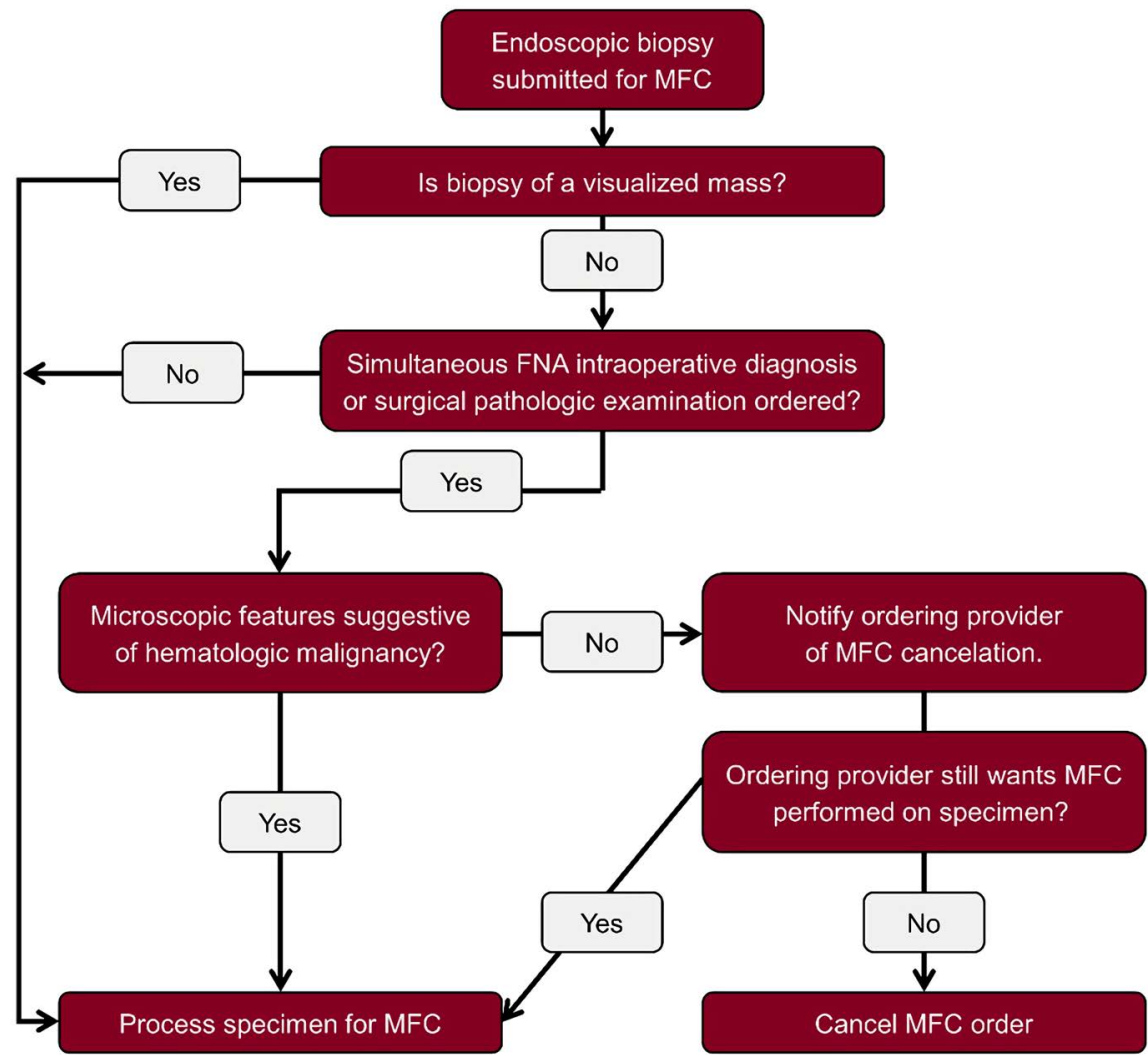

Figure 1. Proposed triaging algorithm. 
slides for the biopsy specimens associated with the MFC study cases. The slides were then reviewed by two senior pathology residents (who each had previously completed five months of hematopathology training) and one board certified hematopathologist. Each reviewer examined the cases in isolation of the other reviewers. Each reviewer was provided with a de-identified synopsis of the endoscopy report and clinical note associated with each endoscopic exam to aid in morphologic assessment, as if they were reviewing each case in real time. After examining the slide and considering the history, each reviewer was given the choice of ordering MFC analysis on the specimen.

\section{Results}

We identified 55 MFC cases corresponding to 32 patients on 33 separate collection dates. Patient and case characteristics are listed in Table 1.

MFC immunophenotyping was initially performed on the biopsy samples using routine protocols that were in place at the time the sample was obtained. Per these protocols, solid tissue specimens such as GI biopsies are disaggregated in a BD Medimachine mechanical disaggregation system [Becton Dickinson (BD, San Jose, $\mathrm{CA})]$ and filtered through a $70 \mu \mathrm{m}$ Falcon filter to produce a tissue cell suspension suitable for immunophenotyping. A cell count is obtained manually via hemocytometer or via a Beckman-Coulter AC-T automated hematology analyzer (Brea, CA). The cell concentration is then adjusted to ensure optimal antibody staining. Samples were processed by lysing erythrocytes using ammonium chloride followed by antibody staining. All tissue specimens were assessed for viability using 7 aminoactinomycin D (7AAD) when possible. MFC analysis utilized a combination of a nine color $\mathrm{B}$ cell tube, eight color $\mathrm{T}$ cell tube, eight color plasma cell tube, and seven color CLL tube. The B cell tube consisted of CD19 V450, CD45 V500, CD5 peridinin-chlorophyll protein-cyanine 5.5 (PerCP-Cy5.5), CD34 Phycoerythrin-Cy7 (PE-Cy7), CD10 allophycocyanin (APC), CD38 APC-R700, and CD20 APC-H7 (an analog of APC-Cy7). The T cell tube consisted of CD4 V450, CD45 V500, CD56 fluorescein isothiocyanate (FITC), CD7 PE, CD5 PerCP-Cy5.5, CD2 PE-Cy7, CD3 APC, and CD8 APC-H7. The plasma cell tube consisted of CD19 V450, CD45 V500, CD38 FITC, CD138 PerCP-Cy5.5, and kappa APC. The CLL tube consisted of CD19 V450, CD45 V500, CD79b PerCP-Cy5.5, CD5 APC, CD23 FITC, CD49d PE, and CD38 APC-H7 (all from BD). Lambda FITC, kappa PE, and intracytoplasmic lambda PE found within the $\mathrm{B}$ cell and plasma cell tubes were obtained from Dako (Carpinteria, CA). MFC analysis was conducted on the majority of cases using 8-color analysis on the BD FACSCanto II flow cytometer (Beckman Coulter Life Sciences, Indianapolis, IN), however a minority of cases were analyzed after upgrading equipment to 10 color analysis. All cases were analyzed using the Kaluza analysis software (Beckman Coulter Life Sciences). Interpretive reports from these analyses were reviewed for this study.

Twenty-one of the 32 patients had a history of hematologic malignancy $(\mathrm{n}=$ $5)$, solid organ or bone marrow transplant $(n=12)$, or both $(n=4)$. This amounted 
Table 1. Patient and case characteristics.

\section{Patient Demographics}

Number of patients

Male

Age in years (range)

History of hematologic malignancy or transplant

\section{MFC Case Demographics}

Total number of MFC cases

Specimen location

- Stomach

- Number positive or suggestive of lymphoma

- Most common endoscopic finding

- Small intestine

- Number positive or suggestive of lymphoma

- Most common endoscopic finding

- Colon

- Number positive or suggestive of lymphoma

- Most common endoscopic finding
32

$65 \%$ of patients

$29-74$

$66 \%$

55

$12(22 \%)$

1

Ulceration

$22(40 \%)$

1

Ulceration

$21(38 \%)$

6

Ulceration (2), Mass (4)

Morphologic Diagnoses of Cases on Which MFC Was Performed (\% of Cases)

- No abnormality

$23.6 \%$

- Non-specific inflammation

- Inflammatory bowel disease

$16.3 \%$

- Infection

$3.6 \%$

- Other diagnostic inflammatory condition*

- Carcinoma

- Lymphoma

- Suggestive of lymphoma

- No morphologic diagnosis rendered

Endoscopic Findings (Number of Cases) ${ }^{* *}$

- Stomach

- Inflammation ${ }^{* * *}$

- Ulceration

8

- Normal

4

- Small bowel

- Inflammation ${ }^{* * *}$

- Ulceration 10

- Mucosal atrophy 1

- White plaques 1

- Normal 7

- Colon

- Inflammation ${ }^{* * *} 3$

- Ulceration 8

- Polyp 1

- Nodules 3

- Stricture 2

- Friability 2

- Normal 3

- Mass 4

${ }^{*}$ Category includes reactive gastropathy, peptic duodenitis, GVHD, drug toxicity; ${ }^{* *}$ some biopsies are from areas of the GI tract with more than one of the above listed features; $* * *$ inflammation is characterized by a combination of the following findings: erythema, congestion, altered vascularity. 
to 28 cases $(60 \%)$ among the MFC-negative group and $5(63 \%)$ among the MFC-positive group. Therefore, recurrent lymphoma and/or post-transplant lymphoproliferative disorder were often diagnostic considerations at the time of endoscopy. For the remaining patients, MFC was ordered by the provider based on a combination of clinical, endoscopic, and radiographic findings. Seventy-one percent of patients for whom MFC was indicative or suggestive of lymphoproliferative disorder had a history of hematologic malignancy and/or transplant, whereas $64 \%$ of patients for whom MFC showed no evidence of lymphoproliferative disorder had such history.

Eight of the 55 specimens (15\%) were diagnostic or suggestive of a lymphoproliferative disorder by MFC (Table 2). Two of these specimens came from the same patient. All eight of these cases consisted of material which was diagnostic or suggestive of lymphoproliferative disorder by morphologic examination as well. Of note, endoscopic ultrasound-guided fine needle aspiration rapid onsite

Table 2. Characteristics of patients with flow cytometry positive for or suggestive of a lymphoproliferative disorder.

\begin{tabular}{|c|c|c|c|c|c|c|c|c|}
\hline Age & Sex & $\begin{array}{l}\text { Biopsy } \\
\text { Site }\end{array}$ & $\begin{array}{c}\text { EUS-FNA } \\
\text { ROSE } \\
\text { Performed? }\end{array}$ & $\begin{array}{l}\text { Indication for } \\
\text { Procedure }\end{array}$ & $\begin{array}{l}\text { Endoscopic } \\
\text { Findings }\end{array}$ & $\begin{array}{c}\text { Clinical } \\
\text { Differential } \\
\text { Diagnosis }\end{array}$ & $\begin{array}{c}\text { Associated History/ } \\
\text { Comorbidities }\end{array}$ & Final Diagnosis \\
\hline 62 & M & $\begin{array}{l}\text { Small } \\
\text { Bowel }\end{array}$ & No & $\begin{array}{l}\text { SBO, abnormal imaging } \\
\text { of duodenum suspicious } \\
\text { for tumor }\end{array}$ & Non-bleeding ulcer & $\begin{array}{l}\text { AdCa vs lymphoma } \\
\text { vs infection vs other }\end{array}$ & $\begin{array}{l}\text { Immunosuppression for } \\
\text { lung transplant for IPF, } \\
\text { EBV viremia }\end{array}$ & $\begin{array}{l}\text { Monomorphic PTLD, } \\
\text { DLBCL type, EBV } \\
\text { negative }\end{array}$ \\
\hline 52 & $\mathrm{M}$ & Stomach & No & Melena & $\begin{array}{l}\text { Ulcers and mucosal } \\
\text { induration }\end{array}$ & $\begin{array}{l}\text { H. Pylori vs NSAID } \\
\text { related injury vs } \\
\text { lymphoma }\end{array}$ & $\begin{array}{l}\text { Tobacco use, alcohol abuse, } \\
\text { history of bleeding GI ulcers }\end{array}$ & $\begin{array}{l}\text { Kappa restricted B cells } \\
\text { by MFC. Atypical } \\
\text { symphoid infiltrate by } \\
\text { morphology. (Lost to } \\
\text { follow up.) }\end{array}$ \\
\hline 63 & M & Colon & No & $\begin{array}{l}\text { Diarrhea, bowel wall } \\
\text { thickening on CT }\end{array}$ & Non-bleeding ulcers & $\begin{array}{l}\text { Infection vs } \\
\text { lymphoma }\end{array}$ & $\begin{array}{l}\text { HBV, TB, nodal T-cell } \\
\text { lymphoma, MAC and } \\
\text { fungal pneumonia }\end{array}$ & MEITL \\
\hline 55 & M & Colon & No & $\begin{array}{l}\text { Abnormal PET scan of } \\
\text { GI tract after diagnosis } \\
\text { of CNS DLBCL }\end{array}$ & $\begin{array}{l}\text { Infiltrative, sessile, } \\
\text { ulcerated obstructing } \\
\text { mass }\end{array}$ & Malignancy & $\begin{array}{l}\text { Recently diagnosed CNS } \\
\text { DLBCL, history of CLL }\end{array}$ & DLBCL and CLL \\
\hline 74 & M & Colon & No & Anemia & $\begin{array}{l}\text { Fungating } \\
\text { non-obstructing mass }\end{array}$ & Malignancy & $\begin{array}{l}\text { Immunosuppression for } \\
\text { heart transplant for } \\
\text { cardiomyopathy, EBV } \\
\text { viremia }\end{array}$ & $\begin{array}{l}\text { Monomorphic PTLD, } \\
\text { DLBCL type, EBV } \\
\text { negative }\end{array}$ \\
\hline 67 & M & Colon & Yes & $\begin{array}{l}\text { Fevers, abdominal pain, } \\
\text { anemia, colonic mass, } \\
\text { LAD, and bony lesions } \\
\text { seen on CT }\end{array}$ & $\begin{array}{l}\text { Fungating ulcerated } \\
\text { mass }\end{array}$ & Lymphoma & $\begin{array}{l}\text { Anemia, fevers, abdominal } \\
\text { pain, diverticulosis, bony } \\
\text { lesions due to a kappa } \\
\text { restricted B cell population }\end{array}$ & DLBCL \\
\hline
\end{tabular}

Abbreviations: AdCa_adenocarcinoma; CLL—chronic lymphocytic leukemia; CNS—central nervous system; CT—computed tomography; DLBCL—diffuse large B cell lymphoma; MEITL_monomorphic epitheliotropic intestinal T-cell lymphoma; EBV_Epstein Barr Virus; EUS-FNA ROSE —endoscopic ultrasound guided fine needle aspiration rapid onsite evaluation; GI-gastrointestinal; IPF-idiopathic pulmonary fibrosis; HBV-hepatitis B; LAD_lymphadenopathy; MAC_mycobacterium avium-intracellulare; MCL_mantle cell lymphoma; MFC-multicolor flow cytometry; PET_-positron emission tomography; PTLD—post-transplant lymphoproliferative disorder; SBO—small bowel obstruction; TB—tuberculosis. 
evaluation (EUS-FNA ROSE) was performed on two of the eight positive cases to augment diagnostic yield/utility of performing MFC. Final diagnoses from these patients included monomorphic post-transplant lymphoproliferative disorder diffuse large B cell lymphoma type, monomorphic epitheliotropic intestinal T-cell lymphoma, mantle cell lymphoma, and a kappa restricted B cell population by MFC analysis/atypical lymphoid infiltrate by morphology on a patient lost to follow up. The remaining 47 cases (85\%) were negative for lymphoproliferative disorder by both MFC and morphologic examination, including 13 cases (28\%) that had no diagnostic abnormality by morphologic examination. Final pathologic diagnoses of these cases can be seen in Table 1 .

Of the categorical variables assessed, abnormal findings on imaging-either a detectable GI mass, bowel wall thickening, or increased GI-related PET avidity-showed a statistically significant association with the presence of a neoplastic population detectable by MFC analysis $(\mathrm{p}=0.02)$. History of transplant, malignancy, or immunosuppression was not statistically significant. Five of the eight positive cases (63\%) had an associated abnormal imaging, compared with just nine of the 47 negative cases (19\%). Two of the eight positive cases were acquired by EUS-FNA.

Among endoscopic findings, 11 of the 47 MFC-negative specimens (23\%) and none of the eight positive specimens were taken from areas of the GI tract that had entirely normal endoscopic features. The most common finding among MFC stomach biopsy specimens was inflammation characterized by a combination of erythema, congestion, and altered vascularity. This was seen in eight of 12 gastric biopsy specimens. One of 12 stomach biopsies came from an area with an endoscopically normal appearance. The most common endoscopic finding among MFC small bowel and colon biopsy specimens was erosion/ulceration, seen in 10 of 22 small bowel biopsies and eight of 21 colon biopsies. Seven of 22 small bowel biopsies and three of 21 colon biopsies came from areas which had an endoscopically normal appearance. All but one of these biopsies which came from an endoscopically normal area showed no diagnostic abnormality or non-specific inflammation by microscopy. One specimen was a stomach biopsy, which upon morphologic examination, showed reactive gastropathy.

Of note, the endoscopically acquired GI biopsies often had cellular concentrations suboptimal for MFC analysis. Our lab considers specimens with a total cell count less than 50,000 to be suboptimal for analysis. For specimens with low cellularity, our lab typically omits the 7AAD tube which we would otherwise use to assess viability. Reference texts have cited that ideal specimens possess cell viability of greater than $85 \%$. This is because lower viabilities can lead to inaccurate results or the necessity to apply fewer antibodies, which can hamper one's ability to detect possible neoplastic populations. In our lab, the number of cells needed for test performance is considered dependent upon the clinical scenario from which the specimen is derived. Therefore, it is at the discretion of the pathologist to determine if the specimen is suitable for analysis. For specimens with suboptimal cellularity, a comment about their suboptimal nature is in- 
cluded in the final report. In the current study, cellular viability was assessed on 32 of the 55 MFC specimens, and ranged from 1-88\%, with a median viability of $14 \%$. The fewest number of antibodies applied to a study specimen was eight, while the most applied was 17.

In the second phase of the study, three of the authors retrospectively applied a new testing/triaging algorithm to the study cases (Figure 1). In brief, (additional details in the Methods section) each reviewer was given H\&E slides, an endoscopy report, and relevant history corresponding to each MFC case - reviewers were asked to apply the algorithm and decide whether MFC was indicated. The microscopic slides were available for review for 49 of the 55 cases (89\%), including 41 of the 47 MFC-negative cases and all eight MFC-positive cases. After reviewing the histology and considering the patients' histories and endoscopy reports, at least one of the three reviewers canceled MFC studies for 36 (88\%) of 41 cases which lacked a detectable abnormal lymphoid population by MFC. Of these cases, reviewers' independent percent cancellation ranged from 59\% - 71\% (24 - 29/41 cases). Independent of one another, all three reviewers agreed to cancel MFC testing for 37\% of the negative cases. Likewise, there was independent, yet unanimous agreement among reviewers to pursue MFC testing for all eight positive cases. When reviewing the slide histology and patient history, the reviewers agreed that if the patient had a history of lymphoma or transplant, they were more likely to order MFC studies regardless of any pathology identified on the slide. Similarly, if a reviewer identified what they considered to be dense lymphoid infiltrate on a slide, they reflexively ordered MFC analysis. Overall, the proposed algorithm which made the hematopathologist/senior pathology resident a gatekeeper, could have led to the cancelation of up to 29 of 55 MFC orders if evaluated independently, or up to 36 of 55 MFC orders if a multi-reviewer approach was adopted, thereby decreasing test utilization by up to $67 \%$.

\section{Discussion}

The GI tract is the most common primary site of extra-nodal lymphomas, with the stomach being the most common site for primary GI lymphomas [3]. Predisposing factors for developing GI hematolymphoid malignancy include infection (Helicobacter pylori), celiac sprue, inflammatory bowel disease, and immunodeficiency syndromes. Recipients of solid organ or stem cell transplants are at risk of developing post-transplant lymphoproliferative disorders (PTLDs), which commonly involve the GI tract. Hematolymphoid malignancies more often involve a single site within the GI tract rather than multiple sites.

Clinical presentation of patients with GI hematolymphoid malignancy is nonspecific and varied. Patients with marginal zone lymphoma involving the stomach often present with complaints of dyspepsia, much as they would with gastritis or peptic ulcer disease, whereas patients with GI lymphoma that produces a large mass can present with symptoms of obstruction. Some GI lym- 
phomas, such as diffuse large B cell lymphoma and enteropathy-associated T cell lymphoma, present with mesenteric lymphadenopathy in addition to a luminal mass. Patients with Epstein Barr virus (EBV) related PTLD may have detectable EBV in the peripheral blood [4] [5] [6] [7].

GI hematolymphoid malignancies also lack specific endoscopic features. While there are a few endoscopic findings suggestive of involvement of the GI tract by lymphoma, such as nodularity of the mucosa known as multiple lymphomatous polyposis, the endoscopic appearance of lymphoma can range from multiple erosions, to large polypoid masses, to effacement of mucosa folds. These endoscopic findings significantly overlap with non-lymphomatous processes such as carcinoma, infection, and inflammatory bowel disease [8] [9] [10].

In the current study, this lack of specificity was evident when reviewing the endoscopic findings associated with material submitted by the ordering provider for MFC analysis. The most common endoscopic findings that prompted biopsy for MFC analysis were ulceration and inflammatory changes, characterized by a combination of erythema, congestion and altered vascularity. We were surprised to realize that tissue from normal-appearing portions of the GI tract was commonly submitted for MFC.

To add another layer of difficulty when triaging these specimens, our institution relies on touch preparations of fresh tissue to assess specimen adequacy and morphology to determine the appropriateness of MFC analysis. Unfortunately, endoscopically acquired GI biopsies often fail to touch off in a useful manner. It is our belief that the mucin which naturally lines the mucosal surface of the GI tract interferes with cell adhesion to the slide. Moreover, the lamina propria of the GI tract is home to inflammatory infiltrates in both health and non-lymphomatous disease. Therefore, it can be difficult to assess the potential utility of MFC analysis on sparsely cellular touch preparations.

For these reasons, we propose that a pathology team member play a gatekeeping role in MFC test utilization for GI biopsies. A recent study published by Mesa et al. touched on this very topic, stating that reactive and clonal populations can be identified and differentiated on FNA smears. Some of the smears from their study were created from endoscopically acquired specimens [11]. Therefore, ROSE performed on EUS-FNAs can play a critical step in ensuring appropriate collection for ancillary studies such as MFC analysis. Of note, in our study, two of the cases for which MFC analysis identified a neoplastic population used specimens acquired via EUS-FNA that were augmented by ROSE.

In this study, MFC did not identify a neoplastic population in the majority (85\%) of PEGIBs. We are fortunate to work in an institution that allows for next day morphologic examination of biopsy specimens. By implementing an algorithm (applied retrospectively in this study) that allowed hematopathology staff to examine the associated fixed biopsy specimens, unnecessary MFC testing could have been avoided in up to $88 \%$ of negative cases reviewed. Moreover, hematopathologists and pathology residents proved adequate triagers, identify- 
ing by morphologic examination and history $100 \%$ of the cases from patients that were ultimately diagnosed with a hematolymphoid neoplasm.

Although similar criteria were applied by each reviewer when reviewing the study cases, consensus was not sought among the three reviewers in this study. Independent of one another, all three reviewers agreed to cancel MFC testing for $37 \%$ of negative cases. The algorithm presented in this study was intended for review to be conducted by one resident or hematopathologist. However, in our institution, if a question remains about a case, seeking consensus among colleagues is encouraged and common. We suspect that had we encouraged our reviewers to discuss the cases amongst themselves, the percent unanimous agreement for test cancelation would have been higher.

Overall, the proposed algorithm could have decreased test utilization by up to $67 \%$, which amounts to a total of $\$ 16,000$ saved in lab charges based on average Medicare reimbursement for flow cytometry using the average number of markers in our lab-an average of $\$ 444$ per patient. Moreover, patients for whom multiple biopsies were submitted from a single endoscopic exam could have been spared up to $\$ 2100$ in lab charges. In addition to patient savings, this algorithm would allow the laboratory to better utilize personnel and resources.

However, this study was not without its limitations. Although there were 55 cases analyzed in this study, those cases came from only 32 separate patients. Moreover, slides were available for review for only 49 of the 55 cases. The robustness of this study would have benefitted from a larger study population. We also acknowledge that patient populations and lab practices at every hospital are different. Our hospital is a tertiary care center and a major transplant center. The large number of patients in this study with a history of hematologic malignancy and/or transplant may have skewed the indications for which MFC analyses were ordered and influenced how many MFC cases were indicative or suggestive of lymphoproliferative disorder. Additionally, not every laboratory will be able to implement such a tight triage timeline as was implemented in this study. We are encouraged by the findings of this study that morphologic examination of fresh specimens during EUS-FNA ROSE or permanent sections of PEGIBs prior to MFC analysis can result in better MFC utilization and increased patient savings. We recommend a follow-up study which implements the algorithm proposed herein in a prospective manner to truly evaluate the safety and effectiveness of said algorithm.

\section{Conflicts of Interest}

The authors declare no conflicts of interest regarding the publication of this paper.

\section{References}

[1] Oldaker, T. and Stone, E. (2007) Quality Control and Quality Assurance in Clinical Flow Cytometry. In: Keren, D.E., Ed., Flow Cytometry in Clinical Diagnosis, 4th Edition, American Society of Clinical Oncology, Chicago, 73-106. 
[2] Johansson, U., Bloxham, D., Couzens, S., Jesson, J., Morilla, R., Erber, W., et al., British Committee for Standards in Haematology (2014) Guidelines on the Use of Multicolour Flow Cytometry in the Diagnosis of Haematological Neoplasms. British Journal of Haematology, 165, 455-488. https://doi.org/10.1111/bjh.12789

[3] Ferry, J.A. (2011) Extranodal Lymphomas. Saunders, Philadelphia, 416p.

[4] Hart, M., Thakral, B., Yohe, S., et al. (2014) EBV-Positive Mucocutaneous Ulcer in Organ Transplant Recipients: A Localized Indolent Posttransplant Lymphoproliferative Disorder. The American Journal of Surgical Pathology, 38, 1522-1529. https://doi.org/10.1097/PAS.0000000000000282

[5] Kuo, S.H. and Cheng, A.L. (2013) Helicobacter Pylori and Mucosa-Associated Lymphoid Tissue: What's New. Hematology. American Society of Hematology Education Program, 2013, 109-117. https://doi.org/10.1182/asheducation-2013.1.109

[6] Odze, R.D. and Goldblum, J.R. (2014) Odze and Goldblum Surgical Pathology of the GI Tract, Liver, Biliary Tract and Pancreas, Expert Consult. Saunders, Philadelphia, 1392p.

[7] Swerdlow, S.H., Campo, E., Harris, N.L., Jaffe, E.L., Pileri, S.A., Stein, H., et al. (2017) WHO Classification of Tumours of Haematopoietic and Lymphoid Tissues. Revised 4th edition, IARC, Lyon, $58 \mathrm{p}$.

[8] Emory, T.S. (2000) Atlas of Gastrointestinal Endoscopy \& Endoscopic Biopsies. American Registry of Pathology, Silver Spring, 449p.

[9] Foukas, P.G. and de Leval, L. (2015) Recent Advances in Intestinal Lymphomas. Histopathology, 66, 112-136. https://doi.org/10.1111/his.12596

[10] Yamamoto, S., Nakase, H., Yamashita, K., Matsuura, M., Takada, M., Kawanami, C., et al. (2010) Gastrointestinal Follicular Lymphoma: Review of the Literature. Journal of Gastroenterology, 45, 370-388. https://doi.org/10.1007/s00535-009-0182-Z

[11] Mesa, H., Rawal, A. and Gupta, P. (2018) Diagnosis of Lymphoid Lesions in Limited Samples: a Guide for the General Surgical Pathologist, Cytopathologist, and Cytotechnologist. American Journal of Clinical Pathology, 150, 471-484.

https://doi.org/10.1093/ajcp/aqy077 